\title{
Large Submandibular Sialoliths: A Report of Three Cases
}

\author{
Nezhad $C^{* 1}$, Mehravaran $R^{2}$ and Sharafi $M^{3}$ \\ ${ }^{1}$ Department of Oral and Maxillofacial Surgery, Shiraz University of Medical Sciences, Shiraz, Iran \\ ${ }^{2}$ Assistant professor of oral and maxillofacial surgery, Azad University, Surgeon, Shiraz-Iran \\ ${ }^{3}$ Operating Theater Assistant, Shiraz-Iran
}

${ }^{*}$ Corresponding author: Nezhad C, Department of Oral and Maxillofacial Surgery, Shiraz University of Medical Sciences, Shiraz, Iran, E-mail: nezhadcyrus@yahoo.com

Citation: Nezhad C, Mehravaran R, Sharafi M (2016) Large Submandibular Sialoliths: A Report of Three Cases. J Dent Oral Care Med 2(1): 106. doi: 10.15744/2454-3276.2.106

Received Date: August 28, 2015 Accepted Date: February 08, 2016 Published Date: February 09, 2016

\begin{abstract}
Sialolithiasis is the most common disease of the major salivary glands. Large sialoliths are a rare occurrence in the literature. The typical clinical presentation varies from swelling at meal times to inflammation, pain, tenderness and swelling of the gland; also, bacterial infections can occur when the gland is filled with the stagnant saliva. To the best of our knowledge, this is the first study presenting three large cases of submandibular gland sialoliths. The patients were selected out of 32 cases of submandibular sialoliths from 1992 to 2015. In one case, the sialolith was brittle and was collected through a specific technique, and another one recurred eighteen months later.
\end{abstract}

Keywords: Sialolith; Submandibular gland

\section{Introduction}

Sialolithiasis is a pathologic condition that affects 60 million people per year [1]. Submandibular gland sialolithiasis is the most common (80 to 90\%), followed by parotid gland sialolithiasis (5 to 15\%) [2]. Although the etiology of submandibular sialolith formation is still unknown [1,3] higher mucus content, greater degree of alkalinity, concentration of calcium and phosphate salts, longer duct and saliva flows against gravity are considered as the etiological factors [4,9]. The size of the sialolith usually varies from $1 \mathrm{~mm}$ to less than $10 \mathrm{~mm}$, rarely more than $15 \mathrm{~mm}$ [4]. Giant sialoliths are classified as those exceeding $15 \mathrm{~mm}$ in every dimension [5].

\section{Case reports}

\section{Case 1}

A 26 year old male was referred due to a painful hard mass in the right side of the floor of the mouth for two weeks which exacerbated during meals and subsided for hours. Clinically, a firm tenderness was detected in the submandibular gland. Intraoral exam revealed a palpable hard mass in the right sublingual caruncle; salivation was not remarkable (Figure 1). A lower occlusal radiography showed a cone shaped radiopaque mass in the Wharton's duct close to the midline. A diagnosis of right submandibular sialolith was made; at a subsequent appointment, the sialolith was removed under local anesthesia by mucosal and duct incision and blunt dissection. After removal of the sialolith about 22mm (Figure 2), a tenacious secretion was evacuated from the duct; hence, sour beverages are recommended for increasing the salivation. The patient was symptom-free postoperatively and complete healing of the surgical wound was observed approximately three weeks later (Figure 3).

\section{Case 2}

A 48 year old male was referred by his physician due to severe pain and tenderness in the submandibular region in the right side. A history of intermittent swelling during meals which reduced gradually was reported for six months. The chief complaint was intensive pain for a week and salty discharge from the ostium four days referral. Clinically, tenderness and pain in the submandibular gland area was found during examination. Intraoral examination revealed edema in the sublingual fold and with bimanual palpation a mass of fibrosis tissue was found. A light pressure of the gland and proximal portion of the duct, purulent discharge was evacuated. The vital signs were within normal limits. 


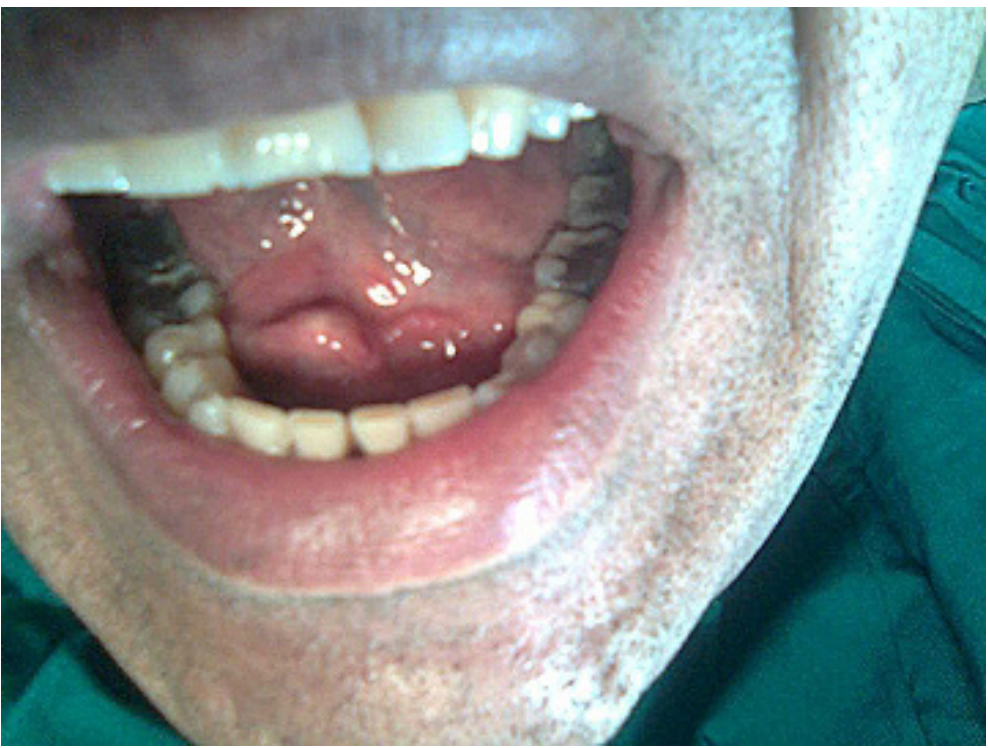

Figure 1: Preoperative intraoral view

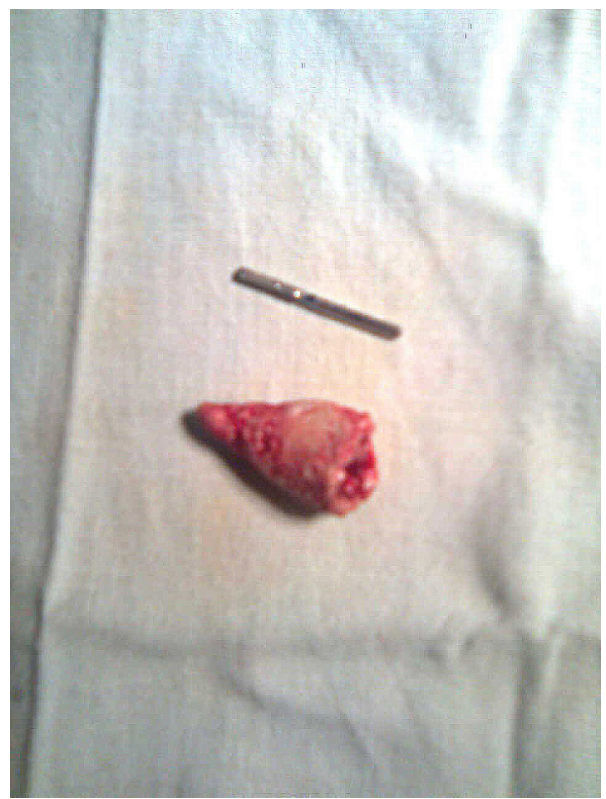

Figure 2: Large submandibular sialolith, $22 \mathrm{~mm}$ in length

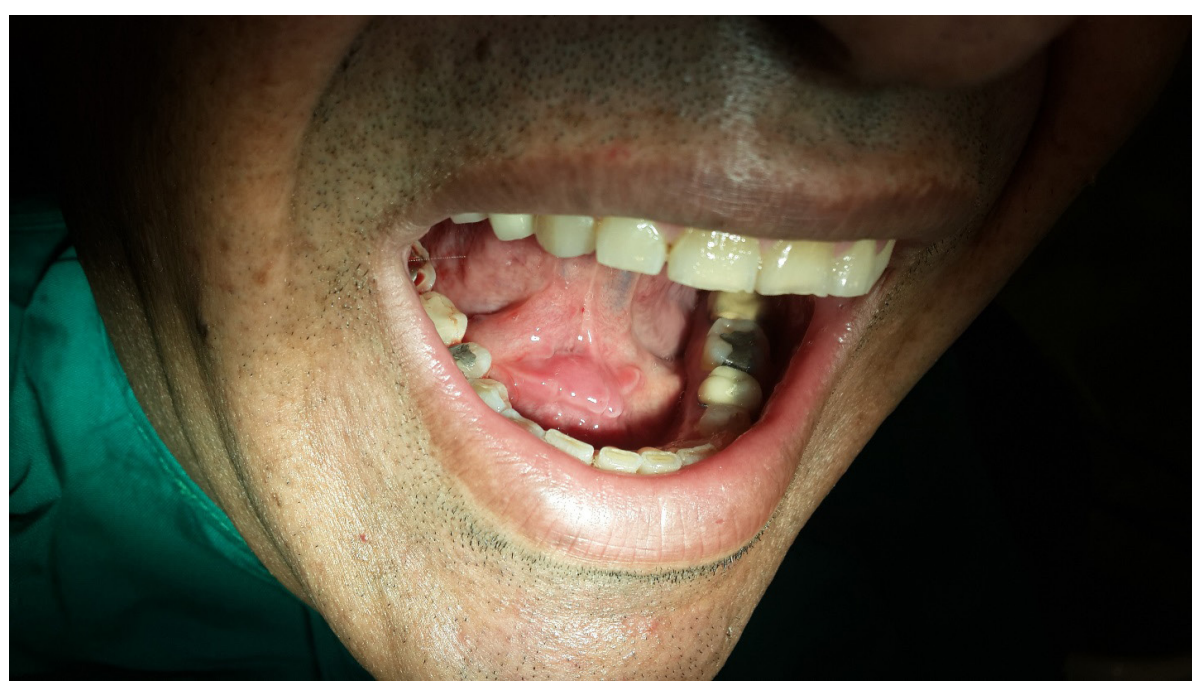

Figure 3: Postoperative view three weeks later 
The radiological examination comprised of occlusal radiography showed a large cubical sialolith in the right Wharton's duct in the vicinity of the premolar- molar region. A $1.5 \mathrm{Cm}$ mucosa and duct incision was made over the mentioned area; through a blunt dissection the sialolith which measured $3.1 \times 28 \times 11 \mathrm{~mm}$ was removed (Figure 4). The pus was discharged, and a copious irrigation done. The patient received antibiotic, analgesic, chlorhexidine and soft diet. The sour sialagogue lozenge beverage was recommended for a week. Three weeks later, the patient was asymptomatic, and the wound was healed anatomically with acceptable salivation. Recurrence occurred close to the right ostium 18 months later and was removed intraorally (Figure 5). Laboratory testing of blood minerals was normal. Limited water intake caused dehydration, as this makes the saliva more concentrated.

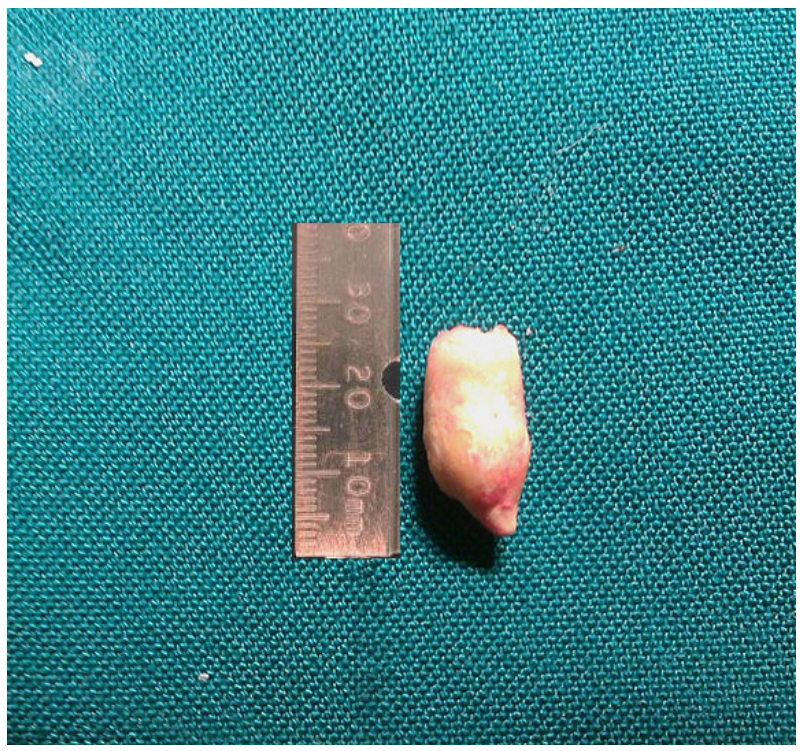

Figure 4: Submandibular sialolith, measuring $3.1 \times 28 \times 11$

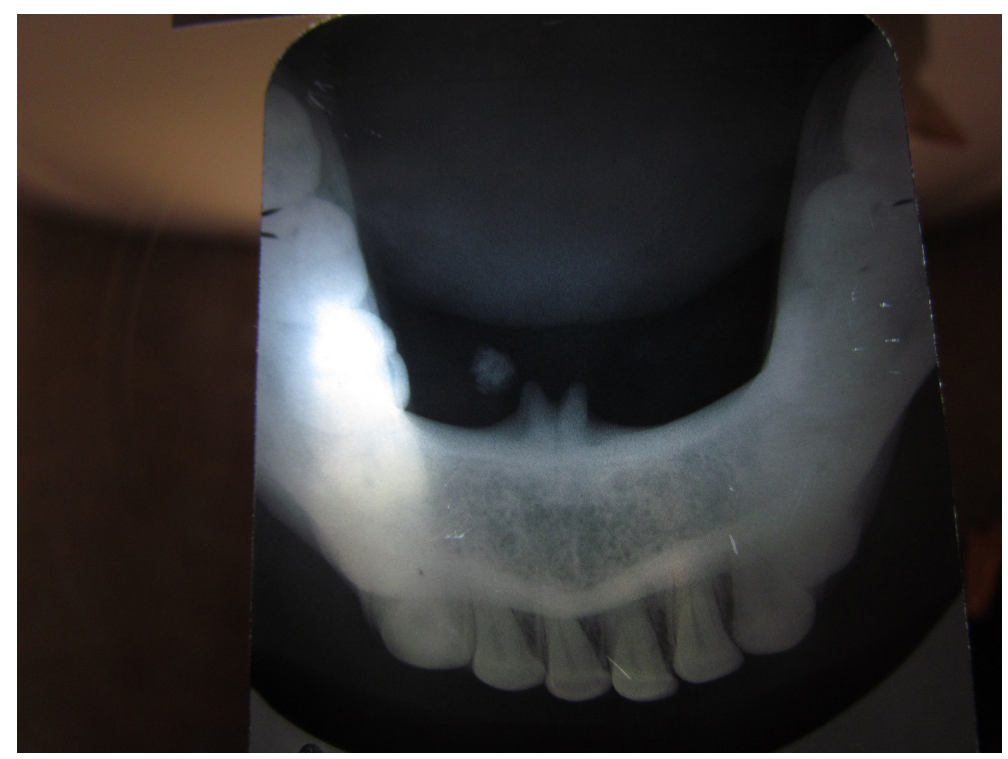

Figure 5: Occlusal radiography, recurred 18 months later

\section{Case 3}

A 41 year-old female visited due to a left lower facial swelling six months ago. She had a history of an intermittent submandibular gland swelling with meals and the symptoms were subsided by time. She refused to undergo surgical intervention and because of her severe anxiety and distress she referred to a psychiatrist for further evaluation. On the basis of cardinal symptoms, the diagnosis of the tomophobia was made. Behavioral psychotherapy as an out-patient treatment was recommended in order to diminish the patient's phobic fears. On re-examination, the submandibular gland was firm and fluctuated. Intraoral bimanual palpation revealed a hard mass in the premolar-molar area. Occlusal radiograph showed a canine shaped radiopaque sialolith about $21 \mathrm{~mm}$ (Figure 6). An 8mm longitudinal incision on the mucosa and blunt dissection Wharton's duct was opened. Because of the brittle sialolith, a traction suture was used in the proximal end of the sialolith to prevent any possibility of the particles being pushed back. All sialolith particles were preserved by bone collector (Figure 7). The patient was asymptomatic following the surgery and complete healing of the surgical site was observed approximately three weeks later. 


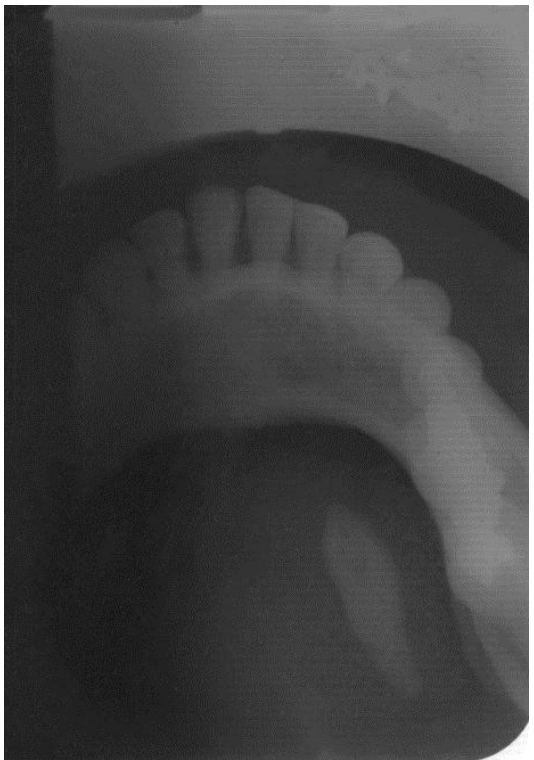

Figure 6: A canine shaped sialolith, $21 \mathrm{~mm}$ in length

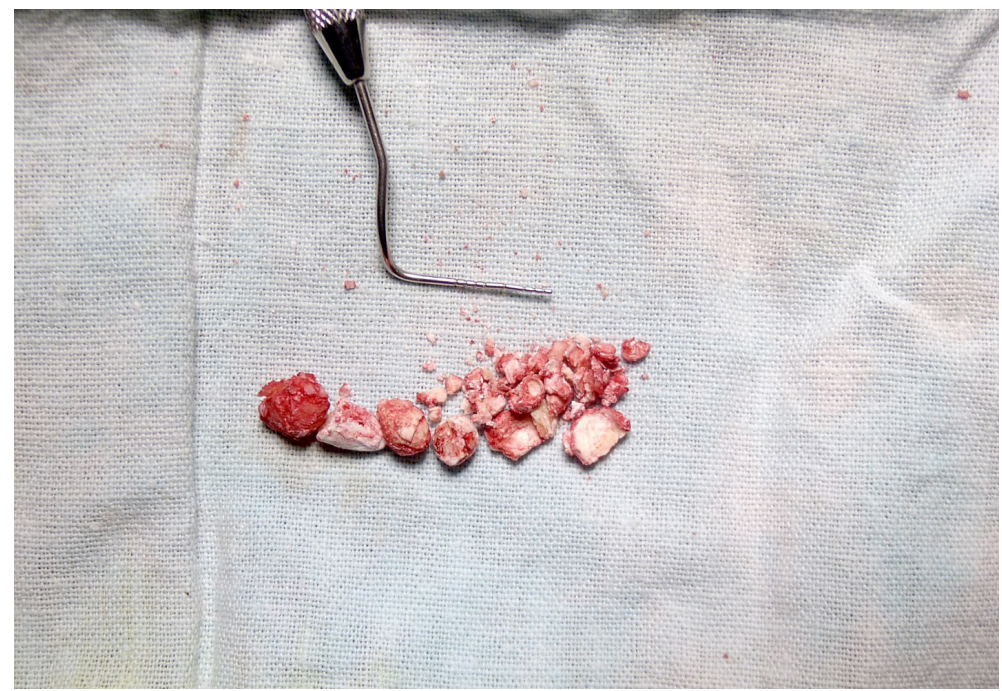

Figure 7: Brittle sialolith

Although large sialoliths have been described in the body of the salivary glands, they are rarely found in the salivary ducts, particularly when the patients have no painful symptoms [5]. Simultaneous sialolithiasis in more than one salivary gland is rare, occurring in fewer than $3 \%$ of cases [6]. Among the diagnostic methods is inspection, palpation, checking the amount of saliva secreted and the identification of a sialolith [7]. Salivary gland stones can occur at any age; yet, they occur most commonly between the third and sixth decades of life [8]. The typical clinical presentation is salivary gland swelling after eating. Persistent swelling causes pain and trismus; untreated sialolithiasis subsequently leads to the salivary gland atrophy or fistula formation [2]. Giant salivary gland calcui is characterized by salivary duct swelling, without any obvious reason or at meal times. Sialolithiasis is associated with swelling, pain, and infection of the affected gland [4]. The most cases present with the classical picture of salivary colic [8].

Current diagnostic imaging tools used in the imaging of salivary stones are: conventional radiography, sialography, ultrasonography, computed tomography, magnetic resonance sialography and sialoendoscopy [10]. Sonopalpation has increased sensitivity and specificity over US alone or physical examination not only for the detection of SMG calculi but also for localization of pathology in the ductal system [11]. The CBCT images showed important data for the evaluation, diagnosis, and treatment plan of the submandibular sialolithiasis [12]. Salivary gland endoscopy is an important diagnostic and therapeutic tool used in the management of sialolithiasis but it must be accompanied by additional techniques to ensure a high rate of stone clearance, symptom resolution, and gland preservation [13]. Treatment options range from a single probing extraction, extraction with sialographic control using the sialoendoscope, LASER intraductal lithotripsy, lithotripsy extracorporeal shock wave (ESWL), to the surgical techniques combining open duct with endoscopic or glandular removal [14]. In some cases, intraoral sialolithotomy is performed when the stone is solitary and easily palpable through the oral cavity [2]. Surgical treatment has traditionally been used as a treatment modality to restore a physiologic salivary flow [4]. Transoral sialolithotomy with sialodochoplasty or sialodenectomy remains as 
the mainstay of treatment [8]. Three submandibular sialoliths involving both the submandibular glands which were removed by intraoral approach and no post-operative complications were noted [6]. Parker and co-workers removed a sialolith in the posterior part of Wharton's duct under general anaesthesia [3].

Based on the present study and previous studies on transoral removal of the calculi by incision in the floor of the mouth, we found this procedure effective, brief and safe for the removal of large, immobile calculi from the hilum of the submandibular gland to the papilla [15]. Transoral removal of the submandibular hilar stones without sialodochoplasty is an effective treatment with good anatomical restoration of the salivary duct and flow [16]. In the future, sialoendoscopy may become one of the main diagnostic and treatment procedures for salivary duct disorders, especially in salivary stone cases [10]. Nowadays, therapeutic options such as interventional sialendoscopy and extracorporeal shock-wave lithotripsy (ESWL) are available in combination with marsupialization or incision of the duct [17]. Sialendoscopy is a reasonable, minimally invasive treatment option for sialolithiasis in order to avoid salivary gland removal. The results of a study indicated that sialendoscopy was the first treatment of choice for submandibular gland sialolithiasis. Complete surgical excision is becoming uncommon as a first-line treatment, but it remains indispensable in certain cases [18]. Phillips and Withrow showed favorable results with the use of intracorporeal holmium laser lithotripsy for the endoscopic management of sialolithiasis with minimal adverse events [19]. The piezoelectric device by tunneling through the glandular channel by the ostium, allows stone fragmentation, with no damage to the surrounding soft tissue [14].

\section{References}

1. Franco A, de Carvalho Mattos MJ, Ferrari F, Dos Reis Neto JM, Carta Gambus LC, et al. (2014) Massive Submandibular Sialolith: Complete Radiographic Registration and Biochemical Analysis through X-Ray Diffraction. Case Rep Surg 659270.

2. Sun YT, Lee KS, Hung SH, Su CH (2014) Sialendoscopy with Holmium: YAG Laser Treatment for Multiple Large Sialolithiases of the Wharton Duct: A Case Report and Literature Review. J Oral Maxillofac Surg 72: 2491-6.

3. Parkar MI, Vora MM, Bhanushali DH (2012) A Large Sialolith Perforating the Wharton's Duct: Review of Literature and a Case Report. J Maxillofac Oral Surg 11: 477-82.

4. Giacomo Oteri, Rosa Maria Procopio, Marco Cicciù (2011) Giant Salivary Gland Calculi (GSGC): Report of Two Cases. Open Dent J 5: 90-5.

5. Gupta A, Rattan D, Gupta R (2013) Giant sialoliths of submandibular gland duct: Report of two cases with unusual shape. Contemp Clin Dent 4: 78-80.

6. Sunder VS, Chakravarthy C, Mikkilinine R, Mahoorkar S (2014) Multiple bilateral submandibular gland sialolithiasis. Niger J Clin Pract 17: 115-8.

7. Filho FB, Martins G, Alves AO, Vieira da Costa JR, Azulay DR, et al. (2014) Rigid swelling of sublingual caruncle area due to the salivary gland duct obstruction by a sialolith. A Bras Dermatol 89: 977-9.

8. Fowell C, MacBean A (2012) Giant salivary calculi of the submandibular gland. J Surg Case Rep 9: 6.

9. Rauso R, Gherardini G, Biondi P, Tartaro G, Colella G (2012) A case of a giant submandibular gland calculus perforating the floor of the mouth. Ear Nose Throat J 91: E25-7.

10. Rzymska-Grala I, Stopa Z, Grala B, Gołębiowski M, Wanyura H, et al. (2010) Salivary gland calculi - contemporary methods of imaging. Pol J Radiol 75: 25-37.

11. Patel NJ, Hashemi S, Joshi AS (2014) Sonopalpation: a novel application of ultrasound for detection of submandibular calculi. Otolaryngol Head Neck Surg 151: 770-5.

12. Meng Y, Laiqing X, Meng J, Na L, Yuanyuan L, et al. (2014) Cone beam CT radiographic diagnosis of submandibular radiopaque sialolithiasis. Hua Xi Kou Qiang Yi Xue Za Zhi 32: 459-63.

13. Zenk J, Koch M, Klintworth N, König B, Konz K, et al. (2012) Sialendoscopy in the diagnosis and treatment of sialolithiasis: a study on more than 1000 patients. Otolaryngol Head Neck Surg. 147: 858-63.

14. Pastor-Ramos V, Cuervo-Díaz A, Aracil-Kessler L (2014) Sialolithiasis. Proposal for a new minimally invasive procedure: Piezoelectric surgery. J Clin Exp Dent 6: e295-8.

15. Juul ML, Wagner N (2014) Objective and subjective outcome in 42 patients after treatment of sialolithiasis by transoral incision of Warthon's duct: a retrospective middle-term follow-up study. Eur Arch Otorhinolaryngol 271: 3059-66.

16. Woo SH, Kim JP, Kim JS, Jeong HS (2014) Anatomical recovery of the duct of the submandibular gland after transoral removal of a hilar stone without sialodochoplasty: evaluation of a phase II clinical trial. Br J Oral Maxillofac Surg 52: 951-6.

17. Vogl TJ, Al-Nawas B, Beutner D, Geisthoff U, Gutinas-Lichius O, et al. (2014) Updated S2K AWMF guideline for the diagnosis and follow-up of obstructive sialadenitis--relevance for radiologic imaging. Rofo 186: 843-6.

18. Matsunobu T, Kurioka T, Miyagawa Y, Araki K, Tamura A, et al. (2014) Minimally invasive surgery of sialolithiasis using sialendoscopy. Auris Nasus Larynx 41: 528-31.

19. Phillips J, Withrow K (2014) Outcomes of Holmium Laser-Assisted Lithotripsy with Sialendoscopy in Treatment of Sialolithiasis. Otolaryngol Head Neck Surg 150: $962-7$. 


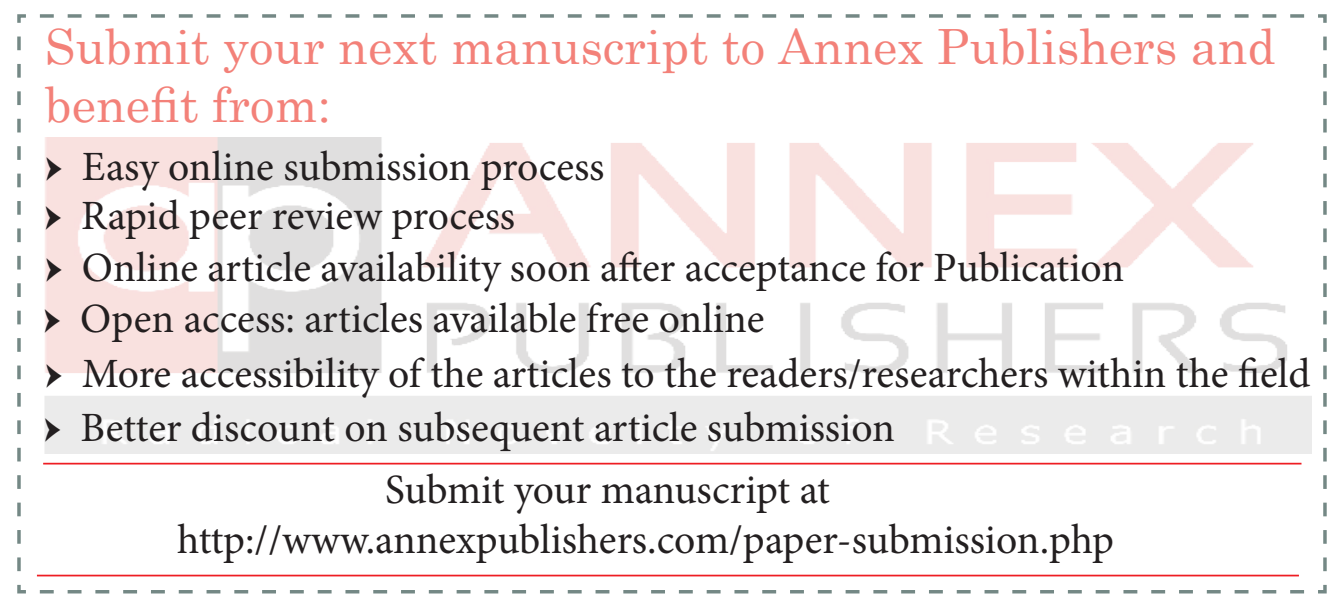

